\title{
Glaucoma surgery with or without adjunctive antiproliferatives in normal tension glaucoma: 2 Visual field progression
}

\author{
W L Membrey, C Bunce, D P Poinoosawmy, F W Fitzke, R A Hitchings
}

\begin{abstract}
Background-Reduction of intraocular pressure by $20-30 \%$ with glaucoma drainage surgery slows disease progression in normal tension glaucoma (NTG). It is not clear whether adjunctive antiproliferative agents are necessary or safe in eyes at low risk for scarring.

Method-61 eyes of 61 white patients with NTG who had undergone a primary guarded fistulising procedure were reviewed. 20 eyes had no antiproliferatives (nil), 29 had peroperative 5-fluorouracil (5-FU), and 12 had peroperative mitomycin C (MMC). Pointwise linear regression analysis (PROGRESSOR for Windows software) was applied to their visual field series starting with the first visual field following surgery and adding subsequent visual fields one at a time. Progression of visual field loss was defined as the appearance of a regression slope $1 \mathrm{~dB}$ per year or more with a significance of $p<0.01$ at one or more visual field locations which remained consistent with the addition of two of three successive visual fields. Time updated covariate analysis was used to determine the relation between variables that changed with time, such as IOP, and the risk of progression.
\end{abstract}

Results-The median percentage IOP reduction was 24.4 for the nil group, 38.0 for the 5-FU group, and 47.5 for the MMC group $(\mathbf{p}=0.001)$. There was a statistically significant relation between percentage change in IOP and risk of visual field progression in the subsequent 6 month period for all patients analysed as one group, hazard ratio $=-0.021 \quad(p=0.002)$. There was a statistically significantly increase in the risk of visual field progression for the MMC group compared with the 5-FU group, hazard ratio $=1.51(p=0.02)$.

Conclusion-In NTG patients, the IOP reduction produced by drainage surgery reduces the risk that visual field progression may be reduced after drainage surgery; this is related to the level of IOP reduction. The percentage drop in IOP during a given time is related to the risk of subsequent visual field progression. However, the use of MMC is associated with a greater risk of visual field progression despite a greater fall in IOP. This visual field deterioration may be related to the functional loss produced by late postoperative complications which have been reported at a higher rate in this group.
The use of adjunctive perioperative 5-FU should maintain a suitable target IOP with preservation of visual function without the additional complications and associated visual deterioration seen with adjunctive MMC.

(Br f Ophthalmol 2001;85:696-701)

Normal tension glaucoma (NTG) has a prevalence of $0.6 \%$ within white populations and is thought to account for $20-30 \%$ of primary open angle glaucoma. ${ }^{1-3}$ It is characterised by an intraocular pressure (IOP) that is found to be within the normal range together with cupping of the optic nerve head and visual field loss of a nerve fibre layer type.

The treatment of progressive NTG represents a therapeutic challenge. The pathogenesis of this condition is thought to be multifactorial. Intraocular pressure plays a part in the continuation of glaucomatous damage. ${ }^{4}$ This has been suggested by the observation that the eye with the higher pressure has the more severe disease ${ }^{56}$ and by interventions that reduce intraocular pressure and slow disease progression. $^{78}$

Treatment for NTG has therefore concentrated on lowering IOP. Because of the risk of failure to achieve low normal IOP in the postoperative period guarded fistulising procedures with or without the use of adjunctive postoperative or perioperative 5 -fluorouracil $(5-\mathrm{FU})^{8}$ or perioperative mitomycin $\mathrm{C}(\mathrm{MMC})^{9}$ have been proposed.

In a previous paper we presented the results of a retrospective review of patients who had undergone fistulising surgery with or without adjunctive antiproliferatives. ${ }^{10}$ We found that the use of adjunctive perioperative 5-FU was associated with an improved IOP outcome compared with no adjunctive antiproliferatives without the higher rate of sight threatening complications and loss of visual acuity associated with the use of adjunctive perioperative MMC.

A more important outcome after filtering surgery is the prevention of further visual field deterioration; however, the detection of "real" progression needs to be differentiated from the inherent "noise" in visual field testing. A number of methods have been used to assess visual field progression. Event analysis is based on specified threshold deterioration compared with baseline field(s), which may be applied to individual visual field locations, clusters, or global measures such as mean deviation. The STATPAC-2 program for the Humphrey field analyser (Humphrey Instruments Inc, San
Accepted for publication 22 December 2000 
Leandro, CA, USA) is a widely used example. Trend analysis looks for a sustained and continued deterioration through all visual fields in the series. Most reported trend analysis has used a model of linear regression. ${ }^{11}{ }^{12}$ This can also be applied to individual points (pointwise linear regression analysis), clusters, or global measures. Pointwise linear regression analysis has been previously described in $\mathrm{NTG}^{13}{ }^{14}$ and shows favourable results compared to STATPAC- $2 .^{15}$

Using pointwise linear regression analysis progressive visual field loss may be detected in $75-84 \%$ of untreated NPG patients within 5 years of follow up. ${ }^{16}$

Glaucoma surgery has been shown to slow the rate of progression in NPG using event type analysis, ${ }^{17}$ linear regression analysis, ${ }^{18}$ and other trend type analysis. ${ }^{19}$ This beneficial effect occurs with IOP reductions of $25-30 \%$ from baseline. ${ }^{17} 18{ }^{20-22}$ However previous reports have generally looked at average IOP reduction during the entire follow up period which is difficult to extrapolate to clinical practice.

The purpose of this study was to investigate the relation between the use of adjunctive antiproliferatives, IOP reduction after filtering surgery, and visual field progression in NPG using a measure of current IOP.

\section{Materials and methods}

Patients attending the normal tension glaucoma clinic at Moorfields Eye Hospital who had been treated with drainage surgery (trabeculectomy) at least 6 months before the time of data collection with at least five postoperative visual field examinations were included in the study. To be classified as normal tension glaucoma patients had to have: (1) glaucomatous cupping of the optic nerve head; (2) reproducible visual field loss of a retinal nerve fibre layer type on static automated perimetry; (3) untreated mean phasing IOP no greater than $21 \mathrm{~mm} \mathrm{Hg}$ and no single reading greater than $23 \mathrm{~mm} \mathrm{Hg}$. The decision to institute treatment had been taken when the patient demonstrated glaucomatous progression using pointwise linear regression analysis (PROGRESSOR $)^{1423}$ of their automated Humphrey 24/2 visual field series.

Patients' hospital records were reviewed and the following data collected: sex; date of birth; race; date of surgery; use of adjunctive antiproliferative agent; median of the mean deviations from the three visual field tests before surgery; preoperative and postoperative IOP; complications following drainage surgery; history of other ocular surgery; and changes in visual acuity.

All non-white patients and patients with a history of previous surgery involving incision of the conjunctiva or penetration of the eye or whose drainage procedure was combined with cataract extraction were excluded.

All patients had undergone guarded fistulising procedure under a limbal or fornix based conjunctival flap. This was done either without the use of adjunctive antiproliferative therapy (designated the nil adjunct group), with the application of $25 \mathrm{mg} / \mathrm{ml} \mathrm{5-FU} \mathrm{for} 5$ minutes to the scleral bed before penetration of the eye (designated the 5-FU group) or with the application of MMC $0.1 \mathrm{mg} / \mathrm{ml}$ for 3-5 minutes to the scleral bed before penetration of the eye (designated the MMC group). The use or otherwise of peroperative antiproliferative agents followed an evolving policy in the NTG clinic. The first series did not receive any, the second received 5-FU, and the third MMC. The operations in all groups were performed by surgeons with varied experience including consultants, fellows, and residents in training at the Glaucoma Unit in Moorfields Eye Hospital.

The patients were seen on a routine basis twice yearly, with more frequent visits during the postoperative period, and as occasion required.

Complications were divided into two groups, perioperative or early postoperative complications and late postoperative or sight threatening complications. Early complications were those occurring in the perioperative or early postoperative period. Late complications were after the initial healing phase had been completed, and were considered to be those seen 3 months or more after surgery. Perioperative or early postoperative complications included: anterior chamber hyphaema; shallowing of the anterior chamber; choroidal effusions; early hypotony (which was defined as an IOP $\leqslant 5 \mathrm{~mm} \mathrm{Hg}$ on two successive occasions at least 4 weeks apart) and early bleb leak.

Late postoperative or sight threatening complications included late bleb leak; infective "blebitis" or endophthalmitis; hypotonous maculopathy, late hypotony, and cataract extraction.

If patients had drainage surgery to both eyes only the first eye to undergo drainage surgery was included in the analysis. This was done because the right and left eyes of an individual patient are known to be interdependent. The median of the IOPs in the 2 years before surgery for each patient was taken as the preoperative baseline. The median IOP and corresponding percentage IOP reduction from the baseline was recorded for each 6 month period postoperatively and was designated as the "current" IOP.

VISUAL FIELD ANALYSIS

The first two visual field tests were ignored in all subsequent analyses to allow for learning.

Pointwise linear regression analysis was applied to the postoperative field series of each of the patients using PROGRESSOR for Windows software. ${ }^{12}$ Any visual field performed before 3 months postoperatively was excluded from the analysis. Progression was defined as the presence of a significant regression slope $(p<0.01)$ showing $1 \mathrm{~dB}$ per year or more of sensitivity loss at the same test location with the addition of two out of three successive field tests to the series starting with the first three. Eyes that showed progression were defined as "visual field failures" and time to failure was taken as the time from surgery to the first of the two out of three successive fields defined above. Eyes were censored after progression had been confirmed. 
Table 1 Demographic data and data on length of follow up, median of MDs closest to date of surgery, median IOP in 2 years preoperatively and number of visual fields by adjunct group. Medians were compared using the Kruskal-Wallis test, categorical data were compared using the $\chi^{2}$ test.

\begin{tabular}{|c|c|c|c|c|}
\hline & Nil adjunct group & 5-FU group & $M M C$ group & $p$ Value $(2 d f)$ \\
\hline Median age at surgery in years (IQ range) & $55.5(49.2-68.0)$ & $68.2(61.4-72.1)$ & $63.8(58.4-73.7)$ & $0.07^{\star}$ \\
\hline Median preoperative follow up in months (IQ range) & $47.2(19.3-89.9)$ & $75.8(28.8-136.4)$ & $60.9(35.8-121.3)$ & $0.15^{\star}$ \\
\hline Median postoperative follow up in months (IQ range) & $90(43.5-112.5)$ & $42(36.0-51.0)$ & $18(12-18)$ & $<0.001^{\star}$ \\
\hline Median of $3 \mathrm{MDs}$ closest to date of surgery (IQ range) & $-14.4(-22.5$ to -10.5$)$ & $-15.4(-20.1$ to -10.3$)$ & $-11.6(-16.7$ to -9.1$)$ & $0.48^{\star}$ \\
\hline Number of left eyes & $13 / 20(65.0 \%)$ & $17 / 29(58.6 \%)$ & $6 / 12(50.0 \%)$ & $0.7 \dagger$ \\
\hline Female & $16 / 20(80.0 \%)$ & $23 / 29(79.3 \%)$ & $8 / 12(66.7 \%)$ & $0.633+$ \\
\hline Median IOP in 2 yearspreoperatively (IQ range) & $16.8(16-19)$ & $17.0(15-19)$ & $15.3(14-17)$ & $0.12^{\star}$ \\
\hline Median number of postoperative visual fields (IQ range) & $21.5(12-24)$ & $11.0(10-13)$ & $6.5(5-9)$ & $<0.001^{\star}$ \\
\hline
\end{tabular}

^Kruskal-Wallis test.

$\dagger \chi^{2}$ test.

Kaplan-Meier plots were constructed and a time dependent Cox model was used to investigate the relation between factors and "visual field failure." These "visual field failure" events were modelled on baseline values for factors that did not change with time, such as sex and adjunct group. However, for factors that did change with time, such as IOP and age, "visual field failure" events were modelled on the values of these risk factors updated for every successive 6 month period. Univariate analyses were first conducted for each factor and those having an effect with a statistical significance of $p<0.1$ were included in the multiple regression model. Categorical outcomes were compared between the three groups using $\chi^{2}$ tests or Fisher's exact test where appropriate. Baseline quantitative data were compared using the Kruskal-Wallis test. Analyses were conducted with spss version 9.05 for Windows and Stata Statistical Software Release 5 (Stata Corporation, USA).

\section{Results}

One hundred and six eyes of 86 patients had drainage surgery 6 or more months before data collection. Six eyes of six patients were excluded because of previous surgery or combined surgery. Six patients were non-white and were therefore excluded. One eye was excluded because the hospital records could not be found. Twelve patients had less than five visual field examinations after surgery. After selection of the first eye where both were eligible this left 61 eyes ( 25 right eyes and 36 left eyes) of 61 patients.

USE OF ADJUNCTIVE ANTIPROLIFERATIVE THERAPY There were 20 eyes $(32.8 \%)$ in the nil adjunct group, 29 eyes $(47.5 \%)$ in the 5 -FU group, and 12 eyes $(19.7 \%)$ in the MMC group. Drainage surgery was performed between May 1986 and July 1992 for the majority of patients in the nil adjunct group (four of 20 patients had surgery after this and therefore overlapped with the other two groups) between November 1992 and
September 1995 in the majority of patients in the 5-FU group (one patient overlapping with the MMC group) and between September 1995 and September 1997 in the MMC group.

Demographic data including median age, median of baseline MDs, number of postoperative visual fields, and preoperative and postoperative follow up are shown for each adjunct group (see Table 1).

IOP AND POSTOPERATIVE COMPLICATIONS

Median IOP in the 2 years before surgery and the percentage fall in IOP over the entire follow up period is shown (see Table 2). We found no evidence of any difference between the groups in the rate of any early or perioperative complications. However, there was a significantly greater proportion of eyes in the MMC group with late hypotony (Table 2).

Two patients in the 5-FU group, one in the MMC group, and none in the nil group underwent cataract extraction after their drainage surgery. There was no significant difference between these figures.

\section{VISUAL FIELD ANALYSIS}

There were 31 "visual field failures"-15 occurring in the nil group, 11 in the $5-\mathrm{FU}$ group, and five in the MMC group.

Univariate Cox regression analysis showed a statistically significant effect of adjunct group on risk of "visual field failure," the 5-FU group having a decreased risk compared with the MMC group (see Fig 1 and Table 3). It also showed a statistically significant decrease in the risk of "visual field failure" with increased percentage fall in "current" IOP from baseline. Figure 2 illustrates this result; it shows a Kaplan-Meier survival plot of "visual field failure" for patients grouped according to whether they had a $30 \%$ reduction in median IOP during the whole follow up period. We found no evidence of an association between "visual field failure" and sex, laterality of eye, age, or mean deviation before surgery (see Table 3 ).

Table 2 Median postoperative IOP and late or sight threatening complications by adjunct group. Medians were compared using the Kruskal-Wallis test, categorical data were compared using Fisher's exact test.

\begin{tabular}{lllll}
\hline & Nil adjunct group & 5-FU group & MMC group & $p$ Value (2 df) \\
\hline $\begin{array}{l}\text { Median percentage reduction in IOP (range) } \\
\text { Late or sight threatening complications }\end{array}$ & $24.4(0-57.9)$ & $38.0(-33.3-64.3)$ & $47.5(22.2-74.2)$ & $0.001^{\star}$ \\
$\quad$ Late bleb leak & $0 / 20(0 \%)$ & $0 / 29(0 \%)$ & $0 / 12(0 \%)$ & $1 \dagger$ \\
Late hypotony & $0 / 20(0 \%)$ & $0 / 29(0 \%)$ & $3 / 12(25 \%)$ & $0.006 \dagger$ \\
Cataract surgery & $0 / 20(0 \%)$ & $2 / 29(6.9 \%)$ & $1 / 12(8.3 \%)$ & $0.461 \dagger$ \\
Endophthalmitis & $0 / 20(0 \%)$ & $0 / 29(0 \%)$ & $1 / 12(8.3 \%)$ & $0.197 \dagger$ \\
Hypotonous maculopathy & $1 / 20(5 \%)$ & $0 / 29(0 \%)$ & $1 / 12(8.3 \%)$ & $0.27 \dagger$ \\
\hline
\end{tabular}

^Kruskal-Wallis test.

†Fisher's exact test. 
Table 3 Estimates of the effect of study factors on time to "visual field failure" from univariate model

\begin{tabular}{lll}
\hline Study factor & Hazard ratio & p Value \\
\hline Adjunct group & & \\
$\quad$ 5-FU & 1.0 & \\
Nil & 1.67 & 0.22 \\
MMC & 3.68 & 0.03 \\
Right eye & 0.95 & 0.89 \\
Female sex & 1.62 & 0.22 \\
Preoperative MD & 1.00 & 0.9 \\
Time updated factors & & \\
$\quad$ "Current" percentage fall in IOP & 0.987 & 0.02 \\
"Current" age & 1.01 & 0.26 \\
\hline
\end{tabular}

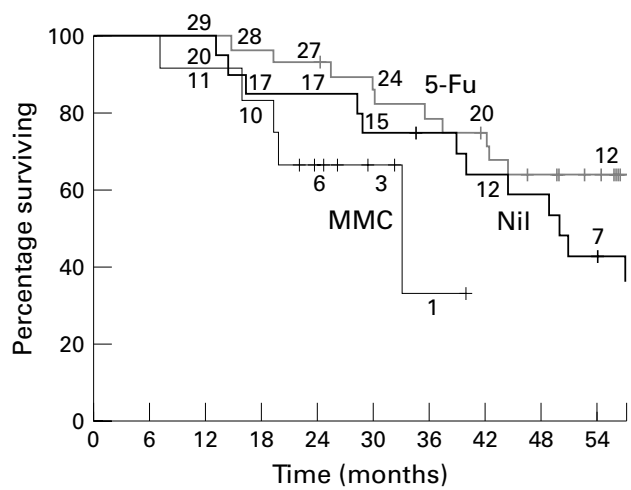

Figure 1 Survival as defined by lack of "visual field failure" from the time of surgery for the three adjunct groups. Marks on survival lines indicate censored data due to end of follow up. Numbers next to survival lines indicate number of patients left in the group at that time.

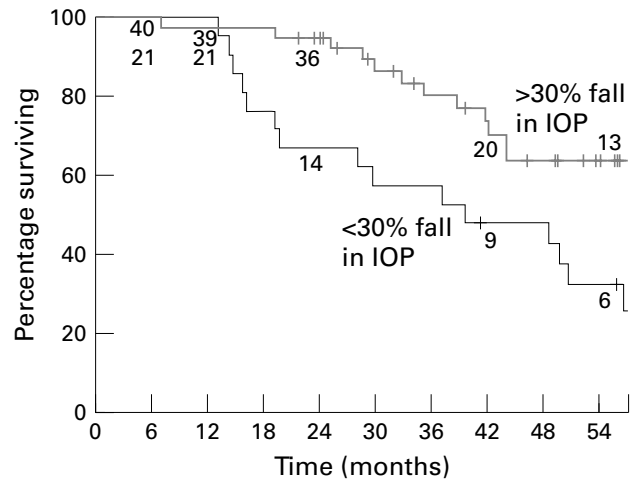

Figure 2 Survival as defined by lack of "visual field failure" from the time of surgery for two groups defined according to a $30 \%$ or more reduction from baseline of their mean postoperative IOP. Marks on survival lines indicate censored data due to end of follow up. Numbers next to survival lines indicate number of patients left in the group at that time.

Table 4 Estimates of the effect of study factors on time to "visual field failure" from multiple variable model

\begin{tabular}{lll}
\hline & Hazard ratio & p Value \\
\hline Adjunct group & & \\
5-FU & 1.0 & \\
Nil & 0.71 & 0.26 \\
MMC & 9.22 & $<0.001$ \\
$\begin{array}{l}\text { Time updated factor } \\
\text { "Current" percentage fall in IOP }\end{array}$ & 0.982 & 0.006 \\
\hline
\end{tabular}

Multiple variable Cox regression analysis showed similar effects of adjunct group and percentage fall in "current" IOP (see Table 4).

A subanalysis was done with the exclusion of all eyes that had late or sight threatening complications, cataract surgery, or a "visual acuity failure" during follow up and this showed very similar results.

\section{Discussion}

At the present time IOP reduction is the only form of treatment for NTG that has been shown to have any long term effect on the course of the disease. ${ }^{71819}$ The reduction needed has to be $20 \%$ or more, ${ }^{172-22}$ the collaborative NTG study accepted an IOP reduction of $20 \%$ or more in their surgically treated arm without further treatment being instituted. Fistulising surgery is likely to play a major part in achieving this end, although some studies have reported IOP reductions of over $30 \%$ with topical therapy and laser trabeculoplasty alone. ${ }^{24}$

In a previous paper we reported on the success of fistulising surgery in NTG patients at reducing IOP and the associated complications. We found that the use of peroperative 5-FU was associated with improved success at reducing IOP by $25 \%$ or more. The use of peroperative MMC was associated with a higher rate of late hypotony and late bleb leak and a worse visual acuity outcome. ${ }^{10}$

This paper reports on the incidence of visual field progression in a similar group of NTG patients who have undergone fistulising surgery. We have again looked at the results for three groups of patients, divided according to the use or otherwise of peroperative antiproliferative agents.

The decision to use peroperative antiproliferatives was largely evolutionary, rather than based on case selection, so that a reasonable comparison can be made between the three groups when assessing the long term results. There was no statistically significant difference between the three groups in preoperative IOP and level of visual field loss as indicated by mean deviation on automated perimetry at the time of surgery.

Considering first this group of NTG patients as a whole, just over $40 \%$ progressed over 4 years, this compares favourably with $60 \%$ progression seen over 4 years in a group of untreated NTG patients using the same progression criteria. ${ }^{16}$ There was a decrease in the risk of visual field deterioration with increasing percentage fall in IOP postoperatively from baseline. Our analysis varies from previous studies in that we looked at IOP updated for each 6 month period postoperatively as a risk factor for visual field progression. Previous studies have either averaged IOP reduction during the whole of the follow up period or have compared groups of patients that have maintaining a specified level of IOP reduction. Our analysis may be more easily extrapolated to clinical practice in that the risk of future visual field progression can be estimated from "current" IOP, taken as the median of readings done in the past 6 months. From our data a "current" IOP that is 30\% less than baseline is associated with a $50 \%$ reduction in the relative risk of subsequent visual field progression compared with a patient who has had no change in IOP. This 
reduction in relative risk is $35 \%$ for a $20 \%$ fall in IOP and $20 \%$ for a $10 \%$ fall in IOP.

Considering now the three adjunct groups, in the univariate model the 5-FU group had a better visual field survival than the nil group. Although this improved survival does not reach statistical significance it might be expected because of the greater median percentage fall in "current" IOP from baseline in the 5-FU group. However the MMC group has the worst visual field survival despite having the greatest fall in IOP, and this is statistically significant when compared with the 5-FU group $(p=0.03)$. The multiple regression model confirms these results, although here the nil group has a better survival than the 5-FU group. Although this is not statistically significant it may also be expected since in this model the effect of adjunct group has been adjusted for the effect IOP.

Progression of glaucoma or the progression of other pathology affecting visual field sensitivity might explain the greater visual field deterioration in the MMC group. A greater rate of glaucomatous visual field progression despite a greater IOP reduction might be explained by differences in baseline factors affecting the risk of progression such as degree of glaucomatous damage. This seems unlikely considering that the three groups were similar in their baseline characteristics.

We have already shown that the use of MMC is associated with a higher rate of both visual acuity loss and hypotonous complications in a larger group of similar patients. ${ }^{10}$ We proposed that the consequences of this hypotony, which includes increased lens opacification, ${ }^{25}$ refractive changes, ocular surface changes, and hypotonous maculopathy might account for the reduction in visual acuity. A higher rate of these complications in the MMC group might also account for the greater rate of visual field progression observed in this group. Although we found no difference in the number of patients undergoing cataract extraction the time periods for the three groups are different and the numbers are too small to make survival analysis possible. Patients may not have been listed for surgery despite functionally significant lens opacification because of the risk of bleb failure after cataract extraction.

Some of these complications would be expected to produce a diffuse reduction in retinal sensitivity ${ }^{2627}$ and therefore a more diffuse type of progression than that caused by glaucoma. It might be possible to distinguish between diffuse and focal progression in these patients by determining the pattern of this progression. ${ }^{28}$

This is a retrospective review of a group of consecutive NPG patients who had undergone fistulising surgery in a single hospital based glaucoma service. Diagnostic criteria for NPG were very similar to that of previous studies. ${ }^{17}$ We included only one eye from each patient in this study and this varies from our previous study, where we only excluded second eyes that were in the same adjunct group. The decision to exclude all second eyes was made because some of the planned analyses were to be conducted on the group as a whole, rather than a comparison between adjunct groups, and inclusion of second eyes might overrepresent risk factors that may be common to both eyes. The criteria for progression in this study is the same as those used by us clinically and have been derived from previous studies comparing pointwise linear regression with STATPAC- 2 change probability analysis in a similar group of patients. ${ }^{12} 141523$ Although we have looked for the effect of potential confounding factors, because of the retrospective nature of this study, unexpected confounding factors cannot be excluded that might provide an alternative explanation for the data. Only a prospective randomised trial can provide definitive evidence, although these results should be useful in guiding the clinical management of these patients until this evidence becomes available.

Surgery remains a good therapeutic option for the patient with disease progression at a rate likely to cause significant functional loss of vision in their lifetime. The greater the percentage reduction in IOP at any time after drainage surgery the lower the risk of subsequent visual field progression. However, the use of MMC in these patients, who are at low risk of scaring, is associated with a higher rate of late hypotonous complications. This may be related to the greater visual acuity loss and visual field progression also seen in this group. We would therefore recommend the use of peroperative 5-FU where fistulising surgery is indicated in similar NTG patients as it was associated with a better IOP outcome without the additional complications seen with MMC.

The International Glaucoma Association supported this project.

1 Bonomi L, Marchini G, Marraffa M, et al. Prevalence of glaucoma and intraocular pressure distribution in a defined population. The Egna-Neumarkt Study. Ophthalmology 1998;105:209-15.

2 Coffey M, Reidy A, Wormald R, et al. Prevalence of glaucoma in the west of Ireland. Br f Ophthalmol 1993;77:
glaucoma glaucoma

3 Dielemans I, Vingerling J, Wolfs R, et al. The prevalence of primary open-angle glaucoma in a population-based study in the Netherlands. The Rotterdam Study. Ophthalmology 1994;101:1851-5

4 Araie $M$, Sekine $M$, Suzuki $Y$, et al. Factors contributing to the progression of visual-field damage in eyes with normaltension glaucoma. Ophthalmology 1994;101:1440-4.

5 Choplin NT. Correlation of asymmetric damage with asymmetric intraocular-pressure in normal-tension glaucoma (low-tension glaucoma). Arch Ophthalmol 1989;107:167-8.

6 Cartwright MJ, Anderson DR. Correlation of asymmetric damage with asymmetric intraocular-pressure in normaltension glaucoma (low-tension glaucoma). Arch Ophthalmol 1988;106:898-900.

7 Abedin S, Simmons RJ, Grant WM. Progressive low-tension Abedin S, Simmons RJ, Grant WM. Progressive low-tension
glaucoma: treatment to stop glaucomatous cupping and field loss when these progress despite normal intraocular (n)

8 Wilson RP, Steinmann WC. Use of trabeculectomy with postoperative 5-fluorouracil in patients requiring extremely low intraocular pressure levels to limit further glaucoma progression. Ophthalmology 1991;98:1047-52.

9 Yamamoto $\mathrm{T}$, Ichien $\mathrm{M}$, Suemori-Matsushita $\mathrm{H}$, et al. [Trabeculectomy for normal-tension glaucoma]. Nippon Ganka Gakkai Zasshi-Acta Soc Ophthalmol fap 1994;98: 579-83.

10 Membrey WL, Poinoosawmy DP, Bunce C, et al. Glaucoma surgery with or without adjunctive antiproliferatives in normal tension glaucoma: 1 Intraocular pressure control and complications. Br f Ophthalmol 2000;84:586-90.

11 Smith SD, Katz J, Quigley HA. Analysis of progressive change in automated visual fields in glaucoma. Invest $O p h-$ thalmol Vis Sci 1996;37:1419-28.

12 Fitzke FW, Hitchings RA, Poinoosawmy D, et al. Analysis of visual-field progression in glaucoma. $\mathrm{Br} \mathcal{f}$ Ophthalmol

13 Noureddin BN, Poinoosawmy D, Fitzke FW, et al. Regression analysis of visual field progression in low 
tension glaucoma [see comments]. $\mathrm{Br} \quad \mathcal{F}$ Ophthalmol 1991;75:493-5

14 McNaught AI, Crabb DP, Fitzke FW, et al. Modeling series of visual-fields to detect progression in normal-tension glaucoma. Graefes Arch Clin Exp Ophthalmol 1995;233: $750-5$.

15 McNaught AI, Crabb DP, Fitzke FW, et al. Visual field progression: comparison of Humphrey Statpac2 and pointwise linear regression analysis. Graefes Arch Clin Exp Oph thalmol 1996;234:411-8

16 Membrey WL, Poinoosawmy DP, Bunce C, et al. In normal tension glaucoma, eyes with visual field loss threatening fixation have no greater risk of continued visual field progression than eyes without threat to fixation. Invest Ophthalmol Vis Sci 1999;40:S65.

17 Collaborative Normal-Tension Glaucoma Study Group Comparison of glaucomatous progression between untreated patients with normal-tension glaucoma and patients with therapeutically reduced intraocular pressur

18 Bhandari A, Crabb DP, Poinoosawmy D, et al. Effect of surgery on visual field progression in normal-tension glaugery on visual field progression in norm

coma. Ophthalmology 1997;104:1 $131-7$.
19 Koseki N, Araie M, Shirato S, et al. Effect of trabeculectomy on visual field performance in central 30 degrees field in progressive normal-tension glaucoma. Ophthalmology 1997;104:197-201.
20 De Jong N, Greve EL, Hoyng PF, et al. Results of a filtering procedure in low tension glaucoma. Int Ophthalmol ;13:131-8

21 Hitchings RA, Wu J, Poinoosawmy D, et al. Surgery for normal tension glaucoma [see comments]. Br $\mathcal{F}$ Ophthalmol 1995;79:402-6.

22 Fontana L, Viswanathan AC, Poinooswamy D, et al. Surgery for normal tension glaucoma. Target intraocular pressure and visual field progression. Acta Ophthalmol Scand 1997;75(S224):43-44

23 Viswanathan AC, Fitzke FW, Hitchings RA. Early detection of visual field progression in glaucoma: a comparison of PROGRESSOR and STATPAC 2. Br f Ophthalmol 1997;81: 1037-42.

24 Schulzer M. Intraocular pressure reduction in normaltension glaucoma patients. The Normal Tension Glaucoma Study Group. Ophthalmology 1992;99:1468-70.

25 Vesti E. Development of cataract after trabeculectomy. Acta Ophthalmol Copenh 1993;71:777-81.

26 Lam BL, Alward WL, Kolder HE. Effect of cataract on automated perimetry. Ophthalmology 1991;98:1066-70.

27 Gillies WE, Brooks AM. Effect of lens opacity on the glaucomatous field of vision. Aust NZ f Ophthalmol 1998; 26(Supp1 1):S19-21.

28 Bengtsson B, Lindgren A, Heijl A, et al. Perimetric probability maps to separate change caused by glaucoma from that caused by cataract. Acta Ophthalmol Scand 1997;75:184-8. 